\title{
Diffuse intrinsic pontine gliomas (DIPG) at recurrence: is there a window to test new therapies in some patients?
}

\author{
Geert O. Janssens ${ }^{1} \mathbb{D} \cdot$ Christof M. Kramm ${ }^{2} \cdot$ Andre O. von Bueren $^{3}$
}

Received: 20 March 2018 / Accepted: 12 April 2018 / Published online: 17 April 2018

(c) Springer Science+Business Media, LLC, part of Springer Nature 2018

To the Editor,

We would like to comment on the manuscript entitled: "Diffuse intrinsic pontine gliomas (DIPG) at recurrence: is there a window to test new therapies in some patients?", published March 2018. In order to design early phase clinical trials for DIPG at the time of disease progression, Lobon-Iglesias et al. try to define a subgroup of long-term ( $>3$ months) survivors among a group of 142 patients with DIPG treated at a single institution [1]. On multivariate analysis (two way ANOVA), steroid-independence ( $\geq 2$ months) and favorable Lansky Play Scale (LPS $>50 \%$ ) at disease progression best predicted survival after relapse and hence, patients eligibility for clinical trials. Although significant on univariate analysis, re-irradiation $(n=14)$ or presence of histone H3.1 mutation $(n=20)$ did not improve statistical significance for prediction.

In a recently published European matched-cohort analysis, comprising 70 patients with DIPG and progression $\geq 90$ days from end of upfront radiotherapy, interval to progression and the use of re-irradiation, remained prognostic for survival on multivariate analysis [2]. Re-irradiation was well tolerated while clinical improvement was observed in nearly $80 \%$ of patients. In this study, a risk score (RS) comprising five categories was developed to predict survival from first progression. Median survival ranges from 1.0 month (RS-1) to 6.7 months (RS-5). No information on duration of steroid-independency, LPS or histone H3 mutations was available for this analysis.

Geert O. Janssens

g.o.r.janssens@umcutrecht.nl

1 Department of Radiation Oncology, University Medical Center Utrecht and Princess Maxima Center for Pediatric Oncology, Utrecht, The Netherlands

2 University Hospital Goettingen, Göttingen, Germany

3 Geneva University Hospitals, Geneva, Switzerland
In absence of a clinical trial, and according to SIOP-E recommendations, re-irradiation of children with DIPG at first progression can be considered when eligibility criteria are fulfilled and radio-necrosis after upfront radiotherapy is excluded [2]. Recently, this policy has been adopted by the National Cancer Institute (https://www.cancer.gov/types /brain/hp/child-glioma-treatment-pdq\#section/_55).

Both studies demonstrate that at least four variables can impact survival from progression $[1,2]$. At present time it will be difficult to bypass the role of re-irradiation at the time of disease progression, even in the absence of a randomized control trial, considered unfeasible for ethical, political and financial reasons within the SIOP-E community. Although we fully support the author's statement that new and more efficient treatments in DIPG are needed, we want to express our concerns on the feasibility of new studies in DIPG at the time of progression for the number of reasons mentioned above.

\section{Compliance with ethical standards}

Conflict of interest The authors declare that there is no conflict of interest.

\section{References}

1. Lobon-Iglesias MJ, Giraud G, Castel D et al (2018) Diffuse intrinsic pontine gliomas (DIPG) at recurrence: is there a window to test new therapies in some patients? J Neurooncol 137:111-118

2. Janssens GO, Gandola L, Bolle S et al (2017) Survival benefit for patients with diffuse intrinsic pontine glioma (DIPG) undergoing re-irradiation at first progression: a matched-cohort analysis on behalf of the SIOP-E-HGG/DIPG working group. Eur J Cancer 73:38-47 